\title{
Prostate cancer - the role of magnetic resonance imaging
}

\author{
Ingrid Mocikovaa ${ }^{a}$ Jozef Babela ${ }^{b}$, Vladimir Balaz ${ }^{b}$
}

\begin{abstract}
Background. This article reviews the potential of magnetic resonance imaging (MRI) in prostate cancer diagnosis. Methods. Systematic scan of Pubmed, Ovid, Medline, Elsevier search engines was used, additional information was found through bibliographic review of relevant articles.

Results. Substantial progress has been made in the imaging of prostate cancer in MR imaging, as well as in advanced MR spectroscopy.

Conclusions. MRI is a non-invasive and direct imaging modality useful for cancer staging, therapy response, detection of recurrence and guided biopsy in previous negative biopsies. MRI with 3.0T system, whole-body MRI, dynamic contrast enhanced MRI, diffusion-weighted imaging (DWI) and MR spectroscopy (MRS) have improved tumor staging, assessment of tumor volume, aggressiveness or recurrence. Implementation of endorectal/phased array superficial MRI findings on 1.5 or 3.0T systems into nomograms for prostate pretreatment prediction is warranted. Surface phasedarray coil MRI accurately defines prostate cancer with elevated risk of extraprostatic disease.
\end{abstract}

Key words: magnetic resonance imaging, prostate cancer, advanced MRI

Received: August 22, 2011; Accepted: February 21, 2012; Available online: May 10, 2012

http://dx.doi.org/10.5507/bp.2012.025

${ }^{a}$ Radiology Department, F.D. Roosevelt Faculty Hospital, nam. gen. Svobodu 1, 97517 Banska Bystrica, Slovak Republic

bUrology Clinic, F.D. Roosevelt Faculty Hospital, nam. gen. Svobodu 1, 97517 Banska Bystrica, Slovak Republic

Corresponding author: Ingrid Mocikova, e-mail ingrid.mocikova@gmail.com

\section{INTRODUCTION}

The major goal for prostatic cancer (PC) imaging is more accurate disease characterization through the synthesis of anatomic, functional and molecular imaging information ${ }^{1}$. The high incidence of prostate cancer, combined with earlier detection and downstaging at the time of diagnosis, and slow natural progression and biological heterogeneity of the disease has made its management a complex and controversial issue. Prostate tumours have a slow growth rate and allow for prolonged survival ${ }^{2}$. The downstaging resulting from prostatic serum antigen (PSA) screening has been accompanied by an unfortunate trend of over detection and over treatment of biologically indolent (low-grade, clinically insignificant) disease. Primary therapies with curative intent (surgery or radiation) provide excellent long-term cancer control, but accompanied by a risk of treatment - related morbidity ${ }^{2}$. The real challenge for physicians is to differentiate patients with advanced cancer and increased risk of morbidity/ mortality from those who will not require immediate or possibly delayed and therapeutic intervention ${ }^{2}$. There are a number of clinical parameters and clinical nomograms to help with the choice of treatment. Defining low risk is the value of prostate serum antigen (PSA) less than 10ng/ $\mathrm{mL}$, Gleason score of 6 and lower, T stage of T2a or lower. High risk is define as PSA value of more than $20 \mathrm{ng} / \mathrm{mL}$, Gleason score of 8 or higher or T2c, intermediate risk is defined as PSA of $10-20 \mathrm{ng} / \mathrm{mL}, \mathrm{T} 2 \mathrm{~b}$, Gleason score of 7 . The Partin staging nomogram is based on clinical stage, Gleason score, serum PSA level, nomograms are widely used for giving a prediction of the final pathologic stage, disease recurrence and estimate of biological potential. Other types of nomogram, such as Kattan's nomograms have been developed to predict stage, recurrence, biological potential ${ }^{3}$. Nomograms are limited by the lack of results from imaging studies that could guide interventions to control local disease. A sensitive and specific enough imaging modality is needed to help differentiate between patients with insignificant disease and patients in need of treatment. This might also decrease the need for biopsies or significantly lower the number of biopsy cores needed. Magnetic resonance imaging (MRI) is able to provide detailed anatomical images due to high spatial resolution, superior contrast resolution and multiplanar capability ${ }^{4}$. In the last decade MRI has improved significantly in technology and reader experience. Newer techniques, such as MR spectroscopic imaging (MRSI), dynamic contrastenhanced MRI (DCE-MRI), perfusion MRI, diffusionweighted imaging (DWI), high-field strength scanning, image postprocessing and picture and communication systems (PACS) improved interpretation of MR prostate cancer images. MRI and MRSI can evaluate tumor aggressiveness, SI ratios from T2WI and biochemical data from MRSI correlate with the Gleason score for PC Gleason score. Recent studies demonstrate that MRI derived parameters (apparent diffusion coefficient [ADC] and T2-relaxation time) on $3.0 \mathrm{~T}$ system correlate with prostate tumor cellularity ${ }^{5}$. MRI provides noninvasively cancer staging, its biological potential, treatment planning, therapy response and local recurrence. MRSI identifies prostate cancer by an increased ratio of choline plus poly- 
amines plus creatine to citrate, citrate level is reduced and choline level elevated in prostate cancer. DWI measures the Brownian motion of water molecules in tissues, while increased cellularity restricts water diffusion. ADC derived from DWI, demonstrates reduced ADC values and increased fractional anisotropy (FA) in prostate cancer. DCE-MRI visualises tumor perfusion and capillary wall permeability and hydrostatic pressure ${ }^{6}$.

\section{TUMOR LOCALISATION}

The staging of PC is based on tumor, node and metastasis (TNM) staging. The latest modification was made in 2010 by the American Joint Committee on Cancer (AJCC). Most important is distinguishing between patients with pathologically organ-defined prostate cancer (pT2) with good prognosis from those with non-confined prostate cancer (pT3-4). Once the cancer extends outside the prostate, the chances of cure are substantially diminished $^{2}$. Radical prostatectomy in organ defined prostate cancer seems to have excellent prognosis, as more than $90 \%$ (ref. $^{2}$ ) are free from recurrence at 5 years. Nomogram prediction of organ defined prostate cancer is high with under the receiver operating characteristic (ROC) curve ranging from 0.79 to 0.82 . MR findings augment significantly clinical nomograms in the prediction of PC in the organ defined stage. A prospective study of 27 patients (MRI-MRSI group and staging nomograms) reported MRI had significantly higher standardized discriminant function coefficients than the Partin tables in prediction of organ-defined prostate cancer ${ }^{2}$. MRI criteria for extracapsular extension include a contour deformity with a step-off or angulated margin, an irregular bulge or edge

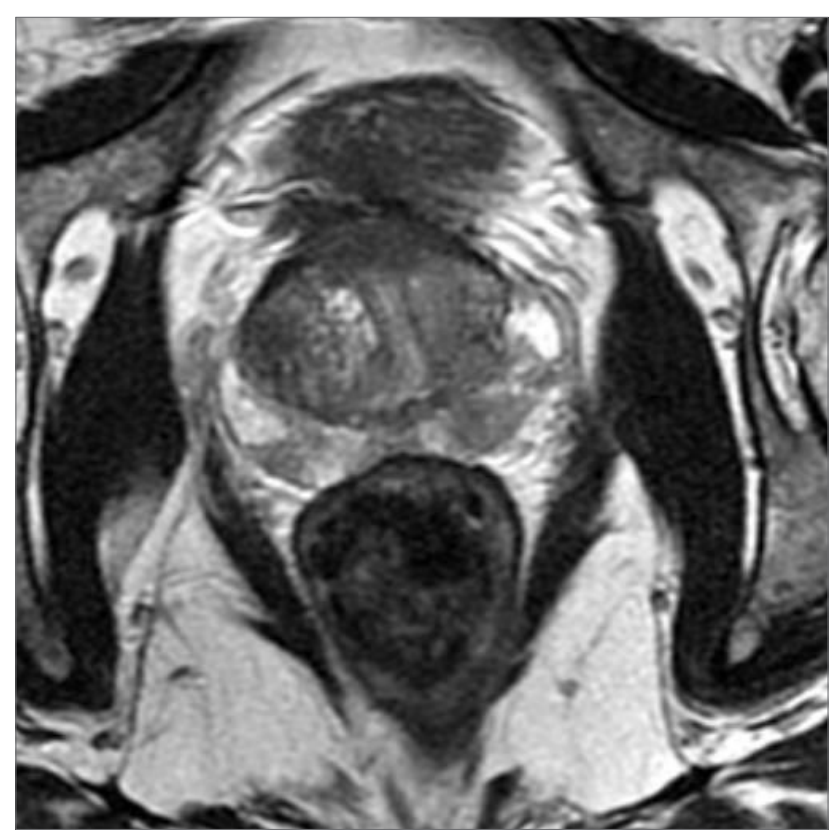

Fig. 1. MRI, axial plane T2WI, bilateral hypointense lesions in peripheral zone - histologically proven prostate cancer, radical prostatectomy with robotic system was done. retraction, direct tumor extension through the capsule, obliteration of the rectoprostatic angle and asymmetry of the neurovascular bundles. MRI findings are significant pre-surgical predictors in patients with extracapsular extension in prostate cancer with augmentative value to clinical variables ${ }^{7}$. MR imaging has been reported to have a $13 \%-95 \%$ sensitivity and $49 \%-97 \%$ specificity for detection of extracapsular extension (ref. ${ }^{1}$ ). The gold standard in 1.5 T MR system for imaging of prostate cancer is dual examination with integrate pelvic phased-array/endorectal phased array. In 3.0T MR the recent advances with faster imaging sequences, more powerful gradient coils, post-processing correction are able to exclude using the endorectal coil, surface phased-array coil is equivalent to endorectal coil in $3.0 \mathrm{~T}$ system $^{8,9}$. However, it is expected, though not yet proven that the use of an endorectal coil at $3.0 \mathrm{~T}$ will allow better prostate cancer detection ${ }^{1}$. A surface phased-array coil MRI accurately stages prostate cancer with elevated risk of extraprostatic disease. This mode of imaging may be reasonable at centres that do not have endorectal coil MRI $\left(\right.$ ref. $^{10}$ ). MRI begins with localisation images, following submillimeter - resolution T2-weighted images (T2WI) in 3 planes for visualizing the anatomy and the architecture of the prostate gland. Prostate cancer has low signal intensity (SI) (Fig. 1), but haemorrhaging, scarring and prostatitis have a similar appearance. This method has a specificity $54-82 \%$ (ref. $^{2}$ ) and sensitivity $46-$ $96 \%$ (ref. $^{2}$ ). A prospective study of 27 patients reported that MRI had significantly higher standardized canonical discriminant function coefficient than Partin tables in predicting extracapsular extension of PC (ref. ${ }^{11}$ ). Detecting peripheral zone $\mathrm{PC}$ has been most effective using MR imaging. Detection of PC in the transition zone is most effective when MR imaging and MR spectroscopic imag-

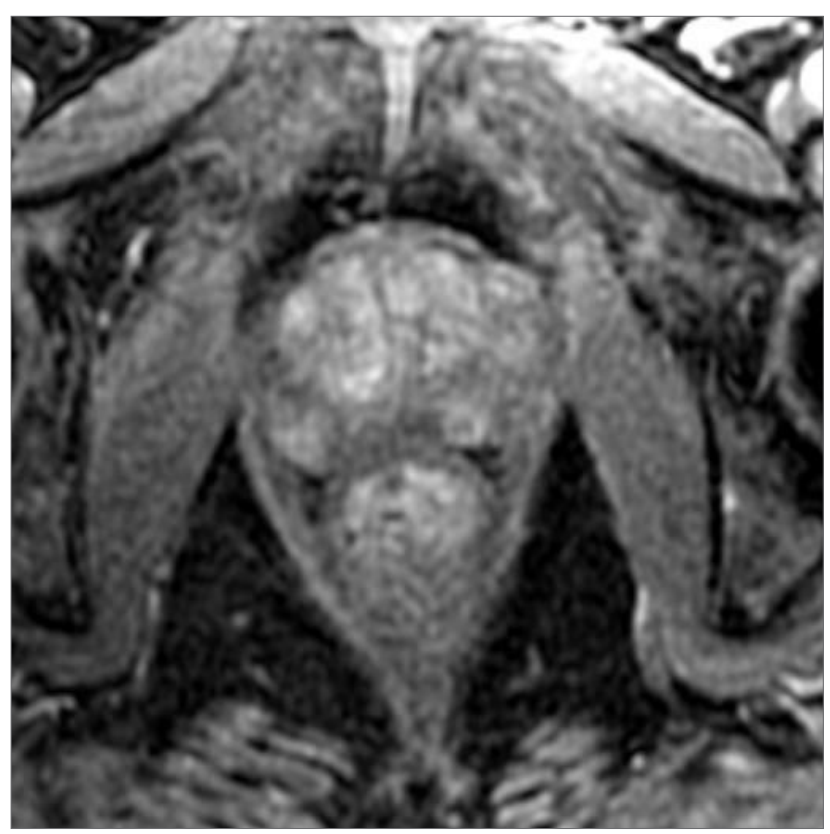

Fig. 2. DCE-MRI, axial plane, bilateral multiple nodular enhancement in early arterial phase - peripheral zone prostate cancer, histologically proven. 
ing are combined. Findings supporting the diagnosis of transition zone tumor are: the presence of homogeneous low-SI region in transition zone and in the absence of dominant peripheral zone tumor. Other alternatives are poorly defined or have speculated lesion margins in transition zone, lack of a low - SI rim (associated with benign adenomatous nodules), interruption of the surgical pseudocapsule, urethral or anterior fibromuscular stromal invasion or lenticular shape.

DWI is a promising method and has recently received attention. Although DWI offers little benefit compared with T2 weighted imaging in the most prostate cancer it appears to be more effective in detection of tumor recurrence after surgery and radiotherapy. Increasing PSA levels with subtle changes in ADC raise suspicious of tumor recurrence. ADC values are found to be negatively correlated with the post-surgical Gleason grade in patients with prostate cancer ${ }^{12}$. Our results show that ADC values might help to predict PC, especially for tumors in the peripheral zone. Given the substantial overlap in the ADC values, the addition of the other MR parameters, such as volumetry and technical improvements in DWI might improve accuracy in the stratification of patient ${ }^{12}$. On DCE-MRI, PC shows early nodular enhancement before the rest of the parenchyma and early washout of contrast medium based on the pathologic microvasculature in the tumor (Fig. 2). These patterns are highly predictive for cancer, but not pathognomic. Some PC are mildly to moderate hypervascular and not detectable with this method. DCE-MRI has been shown to have a sensitivity of $73 \%$ and specificity of $81 \%$, and on the $3.0 \mathrm{~T}$ system a sensitivity of $73 \%$ and specificity of $77 \%$ (ref. ${ }^{1}$ ). Analysis of the dynamic contrast - enhanced MR imaging remains controversial and requires more studies from the various institutions. DWI and the combined technique are more accurate and sensitive than DCE-MRI in the detection of PC. However DWI and DCE-MRI play complementary roles in the accurate detection ${ }^{13}$.

Magnetic resonance spectroscopic imaging (MRSI) measures the metabolite levels in the tumor tissue. Particular metabolites are choline, citrate, creatine and various polyamines. PC causes increased levels of choline, decrease in level of citrate, increased polyamines, but creatine values are normal. The combination of MRSI and MRI can significantly improve tumor localization within the peripheral zone and the detection of extracapsular extension significantly increases the accuracy of less-experienced readers and decreases interobserver variability ${ }^{1}$. Further, new nomograms incorporating MR imaging and MRSI data with clinical variables for predicting of very low-risk cancer are being designed. The diagnostic accuracy of combined $1 \mathrm{H}$ MRSI- DCE-MRI was founf to be higher than PET/CT for identifying local prostate cancer recurrence, mostly in patients with low biochemical progression after radical prostatectomy ${ }^{14}$.

\section{TUMOR VOLUME}

Apart from tumor localisation, tumor volume is helpful as well. Combining different imaging techniques has been shown to significantly increase the accuracy of MRIcalculated tumor volume ${ }^{15,16}$. A safety margin of $5 \mathrm{~mm}$ is considered in prostate cancer.

\section{TUMOR AGGRESSIVNESS}

Accurate grading of the tumor is achievable by biopsy under transrectal ultrasound guidance (TRUS) and assessment of Gleason score. Samplings are usually obtained from 10-12 areas-sextants-peripheral zone: left and apex, left and right mid-gland, left and right base, in addition cores should be obtained from any sonographically suspicious areas. To minimize the need to repeat biopsies many physicians obtain more cores the first time, obtaining 8-10-12 samplings. However, MRI can improve detection of suspected lesions directly before biopsy, in what we call "MRI guidance for prostate biopsies"17. MRI has also been used to guide and monitor ablations, as well as in high-dose-rate brachytherapy rods ${ }^{17}$. MRI has the ability to monitor thermal ablative treatment. Another form is MRI-guided percutaneous interstitial microwave thermoablation in locally recurrent tumor. Chopra et al. showed that using MRI thermometry while applying transurethral MRI guided high intensity-focused ultrasound (HIFU) allowed for stable temperature measurements ${ }^{18}$ to avoid thermal damage of surrounding tissues during the intervention. T2-weighted imaging/DCE- MRI is a promising method for guiding post-HIFU biopsy towards areas containing residual (benign or malignant) viable prostate tissue and to sensitise the detection of locally recurrent cancer in patients with biochemical recurrence. Further research is needed to improve the specificity of the technique and distinction between residual/recurrent cancer and residual benign prostate tissue ${ }^{19}$. MRI is also promising in the detection of tumor with negative biopsy and elevated PSA and this could obviate the need for repeated biopsy. Computed tomography (CT) has no role in newly diagnosed prostate cancer. Its major role is in nodal staging, detection of local and distant metastases, also in cases of local advanced disease- invasion to surrounding structures (pelvic floor, bladder, rectum and pelvic floor muscles).

\section{DETECTION OF SEMINAL VESICLE INVASION}

Seminal vesicle invasion is considered an important predictor of tumor progression and connected with increased risk of lymph node invasion, local tumor recurrence. On MRI T2 weighted images direct signs of seminal vesicle invasion are contiguous low- SI tumor extension from base of the gland to seminal vesicles, focal low- SI within the seminal vesicles disruption or loss of the normal architecture of the seminal vesicles, non-visualization 
or enlarged of the ejaculatory ducts, asymmetric decrease of SI of the seminal vesicles (Fig. 3), obliteration of the angle between the prostate and the seminal vesicles and decreased conspicuity of seminal vesicles. MR imaging has been reported to have $23 \%-80 \%$ sensitivity and $81 \%$ $99 \%\left(\mathrm{ref}^{1}\right)$ specificity for detection of seminal vesicle invasion $^{1}$. Once resection of seminal vesicles during radical prostatectomy has been done, long-term loss of urinary continence had been occurred. Recent postoperative results suggest that preservation of intact seminal vesicles during radical prostatectomy is better for long-term quality of patient's life.

\section{DETECTION OF LYMPH NODES}

The presence of lymph node involvement at the time of primary detection of the prostate cancer appears to be a poor prognostic factor associated with a high probability of progression after treatment. The conventional criterion for detection of metastatic lymph nodes on imaging is a short axis of $8 \mathrm{~mm}^{2}$. MRI and CT have similar efficacy in detecting of lymph node metastases, but equally low sensitivity. Recent studies in so-called MR-lymphography using ultra-small superparamagnetic lymphotropic contrast agents allow detection of metastasis in normal - size lymph nodes, but more clinical studies are necessary $y^{1,2,20}$.

\section{THERAPY RESPONSE}

Early selection of patients who are most likely to benefit from chemotherapy or radiotherapy may prevent the risk of toxicity in non-responding patients with prostate cancer. Early response to chemotherapy is monitored with

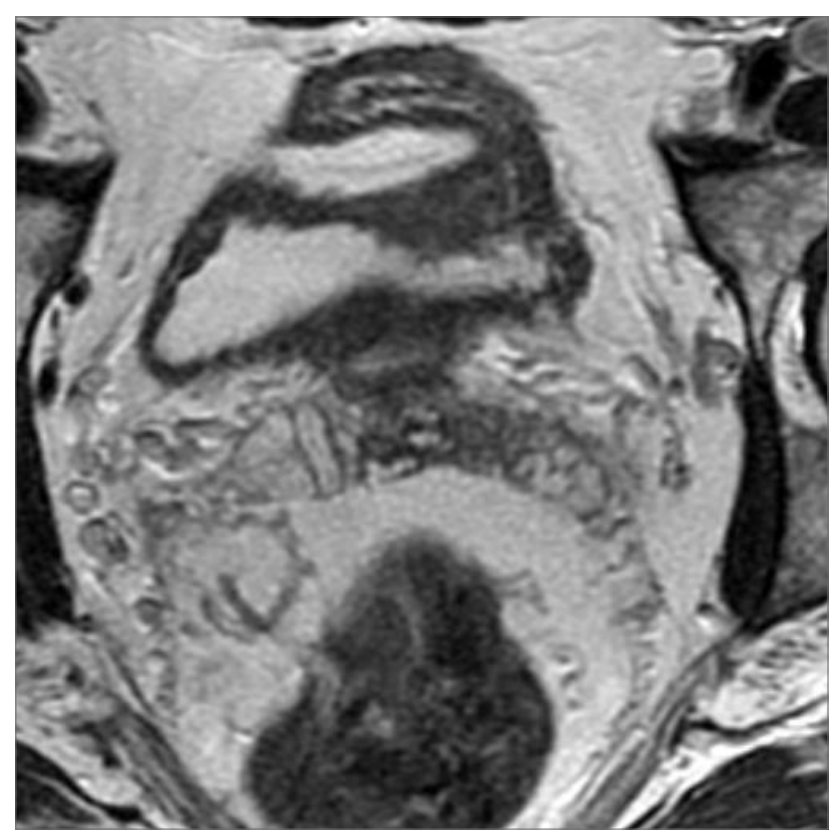

Fig. 3. MRI, sagital plane,T2WI, direct cancer invasion to the seminal vesicles.
DWI especially in bone metastases, as well as significant changes in perfusion due to tumor vascularity and extraction coefficient derived from DCE-MRI ( ref. $^{2}$ ). For identification of local recurrence after radical prostatectomy the combination of MRI and DCE-MRI is an accurate method $^{2}$.

\section{DISCUSSION}

MRI is multipurpose imaging modality. Currently its role has not been exactly defined in clinical guidelines for PC. Patients with suspected prostate cancer and elevated serum markers will undergo an MRI examination and according to the results will either to go on a surveillance protocol, or MRI-guided focal ablation or radical treatment ${ }^{17}$. In patients with abnormal PSA levels and negative biopsy, there is a clinical need for a method with a high sensitivity and a high negative predictive value (unnecessary repeat biopsy could be avoided in patients with negative findings). Poor specificity and positive predictive value are less problematic since obtaining a few additional samples in false-positive areas do not seem to increase the number of biopsy morbidities ${ }^{21}$. MRI as a high-sensitivity high negative predictive value for guiding repeat biopsy towards aggressive cancer foci remains to be determined ${ }^{22}$. DCE-MRI imaging is a key technique for cancer detection with higher sensitivity than that of T2-weighted imaging. In order to achieve the highest possible sensitivity for detection, these two methods need to be combined in one examination. Poor specificity is explained by the presence of a high incidence of inflammation on repeat biopsies. The use of endorectal coil might not be necessary when one is interested mainly in tumor detection and localization $^{8,22}$. Since endorectal coils are expensive, this could have important practical consequences if MRI is to be used for routine screening in patients with elevated PSA and negative prostate biopsy.

MRSI has interesting results in cancer detection and could complement T2-weighted imaging and DCE-MRI imaging. However prostatitis is a cause of false-positive findings for MRSI as well as for DCE-MRI and T2weighted imaging. DWI seems to be superior to DCEMRI and is more accurate and sensitive for detection of prostate cancer. However the combination DWI and DCE-MRI does not significantly improve the accuracy or sensitivity of DWI (ref. ${ }^{22}$ ), but a combination of these methods in one examination are highly recommended.

\section{CONCLUSION}

The high incidence of prostate cancer requires early detection and targeted treatment. Preoperative endorectal MR images significantly improved the accuracy of the surgeon 's decision to preserve or resect the neurovascular bundle at radical prostatectomy. Most cancers are not palpable at the time of primary diagnosis. For this reason MRI in combination with MRSI, DCE-MRI and DWI 
improved direct tumor visualisation. The superior efficacy of MR imaging compared with transrectal ultrasound for the prediction of tumor extraprostatic extension has been shown. There are a number of clinical parameters and nomograms, MRI makes safer, more individualized therapies possible due to high spatial resolution, multiplanar capability and superior contrast resolution. DWI, DCE-MRI, MRSI-MRI are advanced techniques for reflecting tissue biochemistry, vascular tumor feeding, biological potential, local tumor recurrence and planning for biopsies. Pelvic phased-array surface coil in $1.5 \mathrm{~T}$ system performs well in patients with high-risk and select intermediate-risk prostate cancer. It is reasonably accurate in confirming the presence of large tumors in the prostate and detecting extraprostatic extension.

\section{CONFLICT OF INTEREST STATEMENT}

Author's conflict of interest diclosure: The authors stated that there are no conflicts of interest regarding the publication of this article.

\section{REFERENCES}

1. Hricak H, Choyk PL, Eberhardt S, Leibel SA,Scardino PT. Imaging prostate cancer: a multidisciplinary perspective. Radiology 2007;243:2853.

2. Wang L. Incremental value of magnetic resonance imaging in the advanced management of prostate cancer. World J Radiol 2009;31:314.

3. Kattan MW, Scardino PT. Prediction of progression: nomograms of clinical utility. Clin Prostate Cancer 2002;1:90-6.

4. Claus FG, Hricak H, Hattery RR. Pretreatment evaluation of prostate cancer: role of MR imaging and $1 \mathrm{H}$ MR spectroscopy. Radiographics 2004;24:Suppl1:16780.

5. Giusti S, Caramella D, Fruzzetti E, Lazzereschi M, Tognetti A, Bartolozzi C. Peripheral zone prostate cancer. Pre-treatment evaluation with MRI and 3D (1)H MR spectroscopy imaging: correlation with pathologic findings. Abdom Imaging 2010; 35:757-63.

6. Wang L, Van den Bos IC, Hussain SM, Pattynama PM, Vogel MW, Krestin GP. Post-processing of dynamic gadolinium-enhanced magnetic resonance imaging exams of the liver:explanation and potential clinical applications for colour-coded qualitative and quantitative analysis. Acta Radiol 2008;49:6-18.

7. Wang L, Mullerad M, Chen HN, Eberhardt SC, Kattan MW, Scardino PT, Hricak H. Prostate cancer: incremental value of endorectal MR findings for prediction of extracapsular extension. Radiology 2004;232:133-9.

8. Futterer JJ, Engelbrecht RM, Jafer JG,Hartman RP, King BF, Hulsbergen-Van de Kaa CA, Witjes JA,Barentsz JO. Prostate cancer: comparison of local staging accuracy of pelvic phased - array coil versus integrated endorectal-pelvic phased-array coils. Eur Radiol 2007;17:1055-65.
9. Park BK, Kim B, KimCK, Lee HM, Kwon GY. Comparison of Phasedarray 3.0T and endorectal 1.5T magnetic resonance imaging in the evaluation of local staging accuracy for prostate cancer. Comput Assist Tomogr 2007;31(4):534-8.

10. Kim B, Breau RH, Papadatos D, Fergusson D, Doucette $S$, Cagiannos I, Morash C. Diagnostic accuracy of surface coil magnetic resonance imaging at 1.5T for local staging of elevated risk prostate cancer. Can Urol Assoc J 2010;4:257-62.

11. Augustin H, Fritz GA, Ehammer T, Auprich M,Pummer K. Accuracy of 3-Tesla magnetic resonance imaging for the staging of prostate cancer in comparison to the Partin tables. Acta Radiol 2009;50:562-9.

12. Verma S, Rajesh $A$, Morales $H$, Lemen L,Bills $G$, Delworth $M$, Gaitonde $\mathrm{K}$,Yinq J,Samartunga R, Lamba M. Assessment of aggressiveness of prostate cancer: correlation of apparent diffusion coefficient with histologic grade after radical prostatectomy. AJR Am J Roentgenol 2011;196:374-81.

13. Iwazawa J, Mitani T, Sassa S, Shoichi O. Prostate cancer detection with MRI:is dynamic contrast-enhanced imaging necessary in addition to diffusion-weighted imaging? Diagn Interv Radiol. DOI 10.4261/1305-3825.DIR3605-10.1.

14. Panebianco V, Sciarra A, Lisi D, Galati F, Buoconore V, Catalano C, Gentile V, Laghi A, Passariello R. Prostate cancer:1HMRS-DCEMR at $3 T$ versus [(18)] choline PET/CT in the detection of local prostate cancer recurrence in men with biochemical progression after radical retropubic prostatectomy (RRP). Eur J Radiol Feb 2011 [Epub ahead of print] http://dx.doi.org/10.1016/j.ejrad.2011.01.095.

15. Mazaheri Y, Hricak H, Fine SW, Akin O, Shukla-Dave A, Ishill NM Moskowitz CS,Grater JE, Reuter VE, Zakian KL, Touijer KA, Koutcher JA. Prostate tumor volume measurement with combined T2weighted imaging and diffusion-weighted MR: correlation with pathologic tumor volume. Radiology 2009;25:449-57. DOI:10.1148/ radiol. 2523081423

16. Nakashima J,Tanimoto A, Imai Y, Mukai M, Horiguchi Y, Nakagawa $K$, Oya H, Ohigashi T, Marumo K, Murai M. Endorectal MRI for prediction of tumor site, tumor size, and local extension of prostate cancer. Urology 2004;64:101-5. DOI:10.1016/j.urology.2004.02.036.

17. Lawrentschuk $N$, Lindner U. Recent advances in magnetic resonance imaging of prostate cancer. F1000 Med Rep 2010;2:86. DOI:10.3410/ M2-86.

18. Chopra R, Tang K, Butnyk M, Boyes A, Sugar L, Appu S, Klotz L, Bronskill M. Analysis of the spatial and temporal accuracy of heating in the prostate gland using transurethral ultrasound therapy and active MR temperature feedback. Phys Med Biol 2009;54:2615-33. DOI:10.1088/0031-9155/54/9/002.

19. Rouvierer O, Girouin N, Glas L, Ben Cheikh A, Gelet A, Mége Lechevallier F, Rabilloud M, Chapelon JY, Lyonnet D. Prostate cancer transrectal HIFU ablation: detection of local recurrences using T2-weighted and dynamic contrast-enhanced MRI. Eur Radiol 2010;20:48-55. DOI 10.1007/s00330-009-1520-5.

20. Harisinghani MG, Barentz JO, Hahn PF, Deserno WM, Tabataei S, van de KAA CH, de la Rossete J, Weissleder R. Noninvasive detection of clinically occult lymph-node metastases in prostate cancer. N Engl J Med 2003;348:2491-9.

21. Naughton CK, Ornstein DK, Smith DS, Catalona WJ. Pain and morbidity of transrectal ultrasound guided prostate biopsy: a prospective randomized trial of 6 versus 12 cores. J Urol 2000;163:168-71.

22. Cheikh AB, Girouin N, Colombel M, Maréchal JM, Gelet A, Bissery A, Rabilloud $M$, Lyonnet $D$, Rouviére $O$. Evaluation of T2-weighted and dynamic contrast- enhanced MRI in localizing prostate cancer before repeat biopsy. Eur Radiol 2009;19:770-8. DOI: 10.1007/s00330-0081190-8. 